\title{
Nonlinear signal processing in systems with random structure for the case of spatial-time-varying colored Gaussian-Markov noise
}

\author{
Tatiana P. Kolosovskaya \\ Moscow Aviation Institute (National Research University), Moscow, Russia \\ E-mail:mai703@front.ru,kaf703@mai.ru
}

Received 6 September 2017; accepted 7 September 2017

DOI https://doi.org/10.21595/vp.2017.19078

Check for updates

Abstract. The extension of the adaptive estimation theory for nonlinear correlation-extremum systems with random structure to the case when the measurements noise model is a spatial-timevarying Gaussian-Markov colored process is presented and the new signal processing algorithms are derived which provide the system operation in varying and uncertain external conditions.

Keywords: signal processing, filtering, Markov processes, spatial-time-varying colored noise, correlation-extremum methods, optimization of stochastic systems with random structure.

\section{Introduction}

A common practice of much of the work in the area of signal processing in the stochastic dynamic systems carried out in the past has been associated with the state dynamics and measurements modelling with nonlinear (sometimes extremely) functions of the state variables and with corresponding digital filtering algorithms. One of the widely used estimation algorithms in terrestrial and magnetic field navigation systems, in most of the target tracking systems, and in the other areas of applications up to now is an extended Kalman filter (EKF), which performs only relatively robust under operating conditions adequately described in the state and the measurement models, and under natural or artificial disturbances such as abrupt increasing of the measurements noise, informative signal interruption, discontinuous jump of the estimated process features (the last may occur as a result of abrupt target maneuvers), and etc., the behavior of EKF becomes undamped and often ends in diverge.

The causes of the Kalman filter diverge have been first analyzed by Kalman R. E. [1] and Knoll A. [2] and generalized by Sage A. P. and Melse J. L. in [3].

The asymptotic properties of different estimators as a complicated problem is still an open area for further researches.

In most practical applications of filtering theory, the uncertain measurements noise models are sometimes represented by Gaussian processes with unknown probabilistic characteristics (one of the causes of a filter diverge), and the variations of these characteristics and their identification in different conditions play an important role to ensure the stochastic dynamic systems reliability and operation under environment influences. It is specifically necessary to avoid the often-used concept of adding considerable dynamics pseudo-noise to open the filter bandwidth since this tends to veil the differences between the real and the inadequately described models.

An adaptive Kalman filtering algorithm for the standard linear problem under an irregular environment where all variances of zero-mean Gaussian white (system and observation) noises are unknown a priori is obtained in [4] with the modified Kalman gain matrix, which is considered as its "optimal estimate" that depends only on the given data. In [5] an EKF, implemented in radar coordinates, includes together the features based on the introducing model noise into the state estimate covariance matrix before propagation (to increase the robustness of the filter by appropriately distributing the model noise based on the observed trajectory and filter update interval), and on using both static and dynamic model noise terms where the last are adjusted according to the observed track errors and cause the filter to weight new measurements more.

Another limitation of the Kalman filtering schemes (linear or suboptimal extended one) is in the fact that they can process only time-varying signal functions. 
One of the earlier approaches to process the spatial-time-varying signals such as two-dimensional infra-red target images by means of a Kalman filter enhanced by a correlator was proposed in [6].

A further difficulty in the nonlinear signal processing may occur when the measurements models contain colored noises as the covariance matrix is positively semi-definite that has an adverse effect on the filter gain computations.

Some previous work has already dealt with the linear filtering problem for time-varying systems using measurements containing colored time-varying noise, since one of the first researches [7]. The spatial-time-varying filtering problem in the additive spatial-time-varying colored noise has been investigated in [8]. All of these researches refer only to state estimation in the classical stochastic dynamic systems (SDS) with deterministic structure.

Thus, a theoretical investigation was necessary to describe more adequately the SDS and the environment influences on the system to ensure the SDS robustness and operation in different conditions (for example, a tracking system convergence after a target maneuver has occurred).

In [9-11] the correlation-extremum methods were originally applied to signal processing for the systems with random structure and the nonlinear estimation algorithms using spatial-timevarying signals in spatial-time-varying Gaussian white noise (STVGWN) were derived.

In this research, the new estimation algorithms have been derived using a spatial-time-varying measurements model with the spatial-time-varying Gaussian-Markov colored noise (STVGMCN) in conjunction with both nonlinear correlation-extremum system and system with random structure.

\subsection{Problem under consideration}

Consider the following nonlinear estimation problem for the dynamic state process described by a stochastic differential equation (Eq. (1)) [9]:

$\dot{\Lambda}(t)=\mathbf{F}^{(l)}(\boldsymbol{\Lambda}, \mathbf{u}, t)+\mathbf{W}^{(l)}(t), \quad \boldsymbol{\Lambda}\left(t_{0}\right)=\boldsymbol{\Lambda}_{0}, \quad(l=\overline{1, p})$,

where $\boldsymbol{\Lambda}(t)$ is the $n$-dimensional, in general case, state vector, which contains the random, unknown, and time-varying parameters vector $\mathbf{a}^{T}=\left(a_{1}, \ldots, a_{q}\right)$, with initial Gaussian value $\boldsymbol{\Lambda}\left(t_{0}\right), \mathbf{F}^{(l)}(\boldsymbol{\Lambda}, \mathbf{u}, t)$ is the nonlinear deterministic vector function $\mathbf{F}^{(l)}(\boldsymbol{\Lambda}, \mathbf{u}, t)=\left\|f_{i}^{(l)}(\boldsymbol{\Lambda}, \mathbf{u}, t)\right\|$, $(i=\overline{1, n})$ which satisfies Lipschitz conditions, $\mathbf{u}(t)$ is the known control vector, which may depends on the state vector estimates components, $l(t)$ is a stationary Markov process taking values in the set $\{1,2, \ldots, p\}$ (number of the state). Here $\mathbf{W}^{(l)}(t)$ is a vector process of the state Gaussian white noise with diagonal intensity matrix $\mathbf{Q}^{(l)}(t)=\left\|q_{i}^{(l)}(t)\right\|,(i=\overline{1, n})$.

The following measurement Eq. (2):

$$
\mathbf{r}(x, y, t)=\mathbf{S}^{(l)}(x, y, \boldsymbol{\Lambda}, t)+\boldsymbol{\eta}^{(l)}(x, y, t), \quad(l=\overline{1, p}),
$$

describes the observable signal $\mathbf{r}(x, y, t)$ as the $m$-dimensional spatial-time-varying process, where $x, y$ are the space variables - space coordinates at any point $-x \in X=\left[x_{0}, x_{X}\right]$, $y \in Y=\left[y_{0}, y_{Y}\right], t$ is the time variable $t \in T=\left[t_{0}, t_{T}\right], \mathbf{S}^{(l)}(x, y, \boldsymbol{\Lambda}, t)$ is the vector of spatial-time-varying signals of different physical nature, $\boldsymbol{\eta}^{(l)}(x, y, t)$ is the vector process of STVGMCN type.

The spatial-time-varying signal position on the image plane $X O Y$ can be determined by parameters vector $\lambda_{x}(t)=\varphi_{x}(\boldsymbol{\Lambda}, t), \lambda_{y}(t)=\varphi_{y}(\boldsymbol{\Lambda}, t)$. Then the signal may be written as $\mathbf{S}^{(l)}(x, y, \boldsymbol{\Lambda}, t)=\mathbf{S}^{(l)}\left(x-\lambda_{x}, y-\lambda_{y}, t\right)$.

The changes of the structure are assumed to be Markov process with $p$-finite states and the transition intensities $v_{j l}(t)$ and $v_{j}(t)$, where $j, l=\overline{1, p}$. The behavior of the system with random 
structure may be explicated by the example of a tracking system in the cases of a maneuvering target tracking interruption or of great estimation errors, when the target remains in each state for a random period of time, and the Markov process models describe the stochastic continuous process of target dynamics and digital process of the structure changes.

Given the nonlinear estimation problem defined above for Eq. (1-2), we would like to find the finite-dimensional dynamical system whose output is the best minimum variance estimate of the joint Markov process $(\boldsymbol{\Lambda}(t), l(t))^{T}$, for $t \geq 0$, where the suboptimal estimate $\widehat{\Lambda}(t)$ of Markov process $\boldsymbol{\Lambda}(t)$ is the conditional mathematical expectation, and the optimal estimate of discrete process $l(t)$ by the a posteriori probability criterion will be such a value of $l$ that makes the value of the a posteriori probability $\widehat{P}_{l}(t)$ maximum (the $\operatorname{sign}^{\wedge}$ means the a posteriori function value).

\subsection{Solution of the algorithms synthesis and analysis problem}

The solution of the spatial-time-varying signal processing algorithms synthesis and analysis problem presented in this paper is based on a combination of two theories - the correlationextremum systems theory and the theory of stochastic systems with random structure.

The algorithms synthesis for correlation-extremum systems with random structure [9-11] was based on the generalized Fokker-Plank-Kolmogorov-Stratonovich equation for the evolution of joint conditional probability density function of the state dynamics $\boldsymbol{\Lambda}(t)$ and the system structure $l(t)$ given the observed spatial-time-varying data $\mathbf{r}(x, y, t) \omega\left(\boldsymbol{\Lambda}, l, t \mid \mathbf{r}(x, y, \tau), t_{0} \leq \tau \leq t\right)=$ $\widehat{\omega}(\boldsymbol{\Lambda}, l, t)=\widehat{\omega}^{(l)}(\boldsymbol{\Lambda}, t)($ Eq. $(3))$ :

$$
\begin{aligned}
& \frac{\partial \widehat{\omega}^{(l)}(\boldsymbol{\Lambda}, t)}{\partial t}=-\operatorname{div} \widehat{\boldsymbol{\pi}}^{(l)}(\boldsymbol{\Lambda}, t) \\
& -\frac{1}{2} \widehat{\omega}^{(l)}(\boldsymbol{\Lambda}, t)\left[\Phi^{(l)}(\boldsymbol{\Lambda}, \mathbf{r}, t)-\int_{-\infty}^{\infty} \Phi^{(l)}(\mathbf{z}, r, t) \widehat{\omega}^{(l)}(\mathbf{z}, t) d \mathbf{z}\right] \\
& \quad+\sum_{j=1(j \neq l)}^{p} v_{j l}(t) \frac{\hat{P}_{j}(t)}{\hat{P}_{l}(t)}\left[\widehat{\omega}^{(j)}(\boldsymbol{\Lambda}, t)-\widehat{\omega}^{(l)}(\boldsymbol{\Lambda}, t)\right], \quad \omega\left(\boldsymbol{\Lambda}_{0}, t_{0}\right), \quad l=\overline{1, p},
\end{aligned}
$$

where $\hat{P}_{l}(t)$ is the a posteriori probability of the $l$ th state, $\Phi^{(l)}(\boldsymbol{\Lambda}, \mathbf{r}, t)$ is the derivative of the likelihood function logarithm in the $l$ th state $(z$ is the variable, $z \in \boldsymbol{\Lambda})$, $\widehat{\boldsymbol{\pi}}^{(l)}(\boldsymbol{\Lambda}, t)$ is the probability density flow vector in the $l$ th state:

$\widehat{\boldsymbol{\pi}}^{(l)}(\boldsymbol{\Lambda}, t)=\mathbf{K}_{1}^{(l)}(\boldsymbol{\Lambda}, t) \widehat{\omega}^{(l)}(\boldsymbol{\Lambda}, t)-\frac{1}{2} \operatorname{div}\left[\mathbf{K}_{2}^{(l)}(\boldsymbol{\Lambda}, t) \widehat{\omega}^{(l)}(\boldsymbol{\Lambda}, t)\right]$,

where $\mathbf{K}_{1}^{(l)}(\boldsymbol{\Lambda}, t)$ is the local rate vector, $\mathbf{K}_{2}^{(l)}(\boldsymbol{\Lambda}, t)$ is the diffusion matrix, $\omega\left(\boldsymbol{\Lambda}_{0}, t_{0}\right)$ is the initial value of probability density of the state dynamics $\boldsymbol{\Lambda}\left(t_{0}\right)$.

The a posteriori probability density for the whole dynamics process is defined by the following expression $\widehat{\omega}(\boldsymbol{\Lambda}, t)=\sum_{l=1}^{p} \widehat{P}_{l}(t) \widehat{\omega}^{(l)}(\boldsymbol{\Lambda}, t)$. The suboptimal estimate of the state is the probabilistically weighted average $\widehat{\Lambda}(t)=\sum_{l=1}^{p} \hat{P}_{l}(t) \widehat{\Lambda}^{(l)}(t)$.

The presence of the state probability estimate equations (Eq. (4)) (differential or discrete (for a discrete problem statement)) in the estimation algorithms and the relation between these equations are the main distinctive characteristics of signal processing in systems with the random structure $[9,10]$ : 


$$
\begin{aligned}
& \frac{d \hat{P}_{l}(t)}{d t}=-v_{l}(t) \hat{P}_{l}(t)+\sum_{j=1(j \neq l)}^{p} v_{j l}(t) \hat{P}_{j}(t) \\
& \quad+\frac{1}{2} \hat{P}_{l}(t)\left\{\int_{-\infty}^{\infty} \Phi^{(l)}(\mathbf{z}, r, t) \widehat{\omega}^{(l)}(\mathbf{z}, t) d \mathbf{z}-\sum_{k=1}^{p} \hat{P}_{k}(t) \int_{-\infty}^{\infty} \Phi^{(k)}(\mathbf{z}, r, t) \widehat{\omega}^{(k)}(\mathbf{z}, t) d \mathbf{z}\right\} .
\end{aligned}
$$

The a priori state probabilities $P_{l}(t)$ are determined according to Kolmogorov equations:

$$
\frac{d P_{l}(t)}{d t}=-v_{l}(t) P_{l}(t)+\sum_{j=1(j \neq l)}^{p} v_{j l}(t) P_{j}(t)
$$

In this research, the principle of the likelihood function maximum for the STVGMCN $\boldsymbol{\eta}(x, y, t)$ is first extended to the systems with random structure.

The solution of the problem in this paper has been obtained in Gaussian approximation of the a posteriori probability density. In the algorithms synthesis, to simplify the derivation, it was supposed that the signal or image position along one of the axes (e.g., in $y$ direction) was known denoting the signal $\mathbf{S}^{(l)}(x, \boldsymbol{\Lambda}, t)=\mathbf{S}^{(l)}\left(x-\lambda_{x}, t\right)$, and the state parameter $\lambda_{x}$ without index $\lambda_{x}=\lambda$. Then the measurement equation (Eq. (2)) takes the form: $\mathbf{r}(x, t)=\mathbf{S}^{(l)}\left(x-\lambda_{x}, t\right)+$ $\eta^{(l)}(x, t),(l=\overline{1, p})$.

The spatial-time first-order filters (shaping filters) with the transfer function $H(q, p)$, $\left(H_{1}(q), q=\partial / \partial x, H_{2}(p), p=\partial / \partial t\right)$ are used to transform the $\operatorname{STVGWN~}^{(l)}(x, t)$ with spectral densities $C_{0}^{(l)}$ in the case of stationary measurements noise (and may be $C_{0}^{(l)}(t)$, in the case of nonstationary noise) to the Gaussian-Markov colored spatial-varying and time-varying noises, correspondingly: $\boldsymbol{\eta}^{(l)}(x, t)=\mathbf{N}^{(l)}(x, t) H(q, p)$, where $\boldsymbol{\eta}^{(l)}(x, t)$ is the exponentially correlated process (STVGMCN) with spatial-time correlation function $B_{\eta}^{(l)}(\Delta x, \Delta t)=$ $B_{0}^{(l)} \exp \{-\alpha \Delta x-\beta \Delta t\}, B_{0}^{(l)}=C_{0}^{(l)} \alpha \beta / 2$, and frequency characteristics: $G\left(U_{x}\right)=\alpha^{2} /\left(U_{x}^{2}+\alpha^{2}\right)$; $G(\omega)=\beta^{2} /\left(\omega^{2}+\beta^{2}\right)$; where $U_{x}$ and $\omega$ are the spatial and temporal frequencies, the values $\alpha$ and $\beta$ determine the spatial and temporal correlation intervals;

$$
\boldsymbol{\eta}^{(l)}(x, t)=\left\|\eta^{(l)}\left(x, t_{s}\right) \quad \eta^{(l)}\left(x_{k}, t\right)\right\|^{T}=\left\|N^{(l)}\left(x, t_{s}\right) H_{1}(q) N^{(l)}\left(x_{k}, t\right) H_{2}(p)\right\|^{T} \text {, where }
$$
the components $\eta^{(l)}\left(x, t_{s}\right)$ and $\eta^{(l)}\left(x_{k}, t\right)$ are formed in the cuts of the $\eta^{(l)}(x, t)$ process by orthogonal planes for $t=t_{s}$ and $x=x_{k},(k, s=\overline{1, M})$ assuming the spatial and time processes regenerations (or transformations) to be independent.

The STVGMCN model is represented by two components of the time-varying $\eta^{(l)}\left(x_{k}, t\right)$ and spatial-varying $\eta^{(l)}\left(x, t_{s}\right)$ narrowband background described by the Langevin first order differential equations (Eq. (5)), first written for the systems with random structure:

$$
\begin{aligned}
& \frac{\partial \eta^{(l)}\left(x, t_{s}\right)}{\partial x}=-\alpha \eta^{(l)}\left(x, t_{s}\right)+N^{(l)}\left(x, t_{s}\right), \\
& \frac{\partial \eta^{(l)}\left(x_{k}, t\right)}{\partial t}=-\beta \eta^{(l)}\left(x_{k}, t\right)+N^{(l)}\left(x_{k}, t\right), \quad k, s=\overline{1, M} .
\end{aligned}
$$

For the case when the structure changes represent Markov process with two states $(l=1,2)$ and the transition intensity $v(t)$ the new solution of nonlinear filtering problem in STVGMCN for systems with random structure has been obtained in the form of the following correlationextremum algorithms for computing the a posteriori probabilities of state (Eq. (6)), the state estimates (Eq. (7)), and the covariance (Eq. (8)).

The differential equation for the a posteriori probabilities of state is presented below: 


$$
\begin{aligned}
& \frac{d \hat{P}_{1}(t)}{d t}=-\left\{v-\frac{1-\hat{P}_{1}(t)}{2 C_{X}^{(1)}}\left(2\left[k_{1}+B^{(1)}\left(\Delta \lambda_{(1)}\right)\right]-\frac{1}{\alpha^{2}}\left[B_{x}^{(1)}\left(\Delta \lambda_{(1)}\right)+k_{x}^{(1)}\right]\right.\right. \\
& \quad-\frac{1}{\beta^{2}}\left[B_{t}^{(1)}\left(\Delta \lambda_{(1)}\right)+k_{t}^{(1)}\right]-\left[2 \frac{\partial^{2} B^{(1)}\left(\Delta \lambda_{(1)}\right)}{\partial \Delta \lambda_{(1)}^{2}}+\frac{1}{\alpha^{2}} \frac{\partial^{2} B_{x}^{(1)}\left(\Delta \lambda_{(1)}\right)}{\partial \Delta \lambda_{(1)}^{2}}\right. \\
& \left.\left.\left.+\frac{1}{\beta^{2}} \frac{\partial^{2} B_{t}^{(1)}\left(\Delta \lambda_{(1)}\right)}{\partial \Delta \lambda_{(1)}^{2}}\right] \sigma_{(1)}^{2}(t)+n_{x}^{(1)}+n_{t}^{(1)}\right)\right\} \hat{P}_{1}(t) \\
& +\left\{v-\frac{\hat{P}_{1}(t)}{2 C_{X}^{(2)}\left(2\left[k_{2}+B^{(2)}\left(\Delta \lambda_{(2)}\right)\right]-\frac{1}{\alpha^{2}}\left[B_{x}^{(2)}\left(\Delta \lambda_{(2)}\right)+k_{x}^{(2)}\right]\right.}\right. \\
& \quad-\frac{1}{\beta^{2}}\left[B_{t}^{(2)}\left(\Delta \lambda_{(2)}\right)+k_{t}^{(2)}\right]-\left[2 \frac{\partial^{2} B^{(2)}\left(\Delta \lambda_{(2)}\right)}{\partial \Delta \lambda_{(2)}^{2}}+\frac{1}{\alpha^{2}} \frac{\partial^{2} B_{x}^{(2)}\left(\Delta \lambda_{(2)}\right)}{\partial \Delta \lambda_{(2)}^{2}}\right. \\
& \left.\left.\left.\quad+\frac{1}{\beta^{2}} \frac{\partial^{2} B_{t}^{(2)}\left(\Delta \lambda_{(2)}\right)}{\partial \Delta \lambda_{(2)}^{2}}\right] \sigma_{(2)}^{2}(t)+n_{x}^{(2)}+n_{t}^{(2)}\right)\right\}\left[1-\hat{P}_{1}(t)\right], \\
& \hat{P}_{2}(t)=1-\hat{P}_{1}(t),
\end{aligned}
$$

where $\hat{P}_{2}(t)$ is the a posteriori probability of the second state, $\Delta \lambda_{(l)}(t)$ is the state estimate error $\Delta \lambda_{(l)}(t)=\lambda(t)-\hat{\lambda}^{(l)}(t), \sigma_{(l)}^{2}(t)$ is the variance of the a posteriori probability density function $\sigma_{(l)}^{2}(t)=\left\langle\left[\left(\lambda(t)-\hat{\lambda}^{(l)}(t)\right]^{2}\right\rangle,(l=\overline{1,2}) ; B^{(l)}\left(\Delta \lambda_{(l)}, t\right)\right.$ is the spatial correlation function in the $l$ th state $B^{(l)}\left(\Delta \lambda_{(l)}, t\right)=\left\langle\mathbf{S}^{(l)^{T}}\left(x-\hat{\lambda}^{(l)}, t\right) \mathbf{S}^{(l)}(x-\lambda, t)\right\rangle$, (or for the scalar measurement: $\left.B^{(l)}\left(\Delta \lambda_{(l)}, t\right)=\left\langle S^{(l)}\left(x-\hat{\lambda}^{(l)}, t\right) S^{(l)}(x-\lambda, t)\right\rangle\right)$,

$B_{t}^{(l)}\left(\Delta \lambda_{(l)}\right)=\left\langle\frac{\partial S^{(l)}(x-\lambda, t)}{\partial t} \frac{\partial S^{(l)}\left(x-\hat{\lambda}^{(l)}, t\right)}{\partial t}\right\rangle, \quad k_{t}^{(l)}=\int_{-X}^{X}\left[\frac{\partial S^{(l)}\left(x-\hat{\lambda}^{(l)}, t\right)}{\partial t}\right]^{2} d x$,
$B_{x}^{(l)}\left(\Delta \lambda_{(l)}\right)=\left\langle\frac{\partial S^{(l)}(x-\lambda, t)}{\partial x} \frac{\partial S^{(l)}\left(x-\hat{\lambda}^{(l)}, t\right)}{\partial x}\right\rangle, \quad k_{x}^{(l)}=\int_{-X}^{X}\left[\frac{\partial S^{(l)}\left(x-\hat{\lambda}^{(l)}, t\right)}{\partial x}\right]^{2} d x$, $n_{x}^{(l)}=\frac{1}{2 C_{0}^{(l)} \alpha^{2}} \int_{-X}^{X} \frac{\partial \eta^{(l)}(x, t)}{\partial x} \frac{\partial S^{(l)}\left(x-\hat{\lambda}^{(l)}, t\right)}{\partial x} d x$, $n_{t}^{(l)}=\frac{1}{2 C_{0}^{(l)} \beta^{2}} \int_{-X}^{X} \frac{\partial \eta^{(l)}(x, t)}{\partial t} \frac{\partial S^{(l)}\left(x-\hat{\lambda}^{(l)}, t\right)}{\partial t} d x$.

$C_{X}^{(l)}$ is the specific spectral intensity of the $\operatorname{STVGWN~N}^{(l)}(x, t)$ in the $l$ th state, $C_{X}^{(l)}=C_{0}^{(l)} / X$. The equations (Eq. (6)) have been derived using the assumption of the "unpowered" parameters. This assumption means that the integrals $\int_{-X}^{X}\left[S^{(l)}\left(x-\hat{\lambda}^{(l)}, t\right)\right]^{2} d x$ and $\int_{-X}^{X} r^{2}(x, t) d x$, which represent the signal power and are explicitly independent of the estimate parameter, may be included in the $k_{1}$ and $k_{2}$ coefficients, and the integrals of the squared signals derivatives, with respect to $t$ and $x$, may be involved in the $k_{t}^{(l)}$ and $k_{x}^{(l)}$ terms.

The estimates $\hat{\lambda}^{(l)}(t)$ and covariances $\sigma_{(l)}^{2}(t)$ in each state are combined to obtain the suboptimal (as the system is nonlinear) estimate $\hat{\lambda}(t)$ and covariance $\sigma^{2}(t)$ for the whole process by using a weighted sum, where the weighting factor is the a posteriori probabilities of states $\hat{P}_{l}(t)$.

Using the obtained derivatives of the likelihood function logarithm with respect to the estimates in each state and some rearrangements, the following state estimate equation (Eq. (7)) 
has been derived:

$$
\begin{aligned}
& \frac{d \hat{\lambda}^{(l)}(t)}{d t}=f^{(l)}\left(\hat{\lambda}^{(l)}, u, t\right)-\frac{\sigma_{(l)}^{2}(t)}{2 C_{X}^{(l)}}\left[2 \frac{\partial B^{(l)}\left(\Delta \lambda_{(l)}, t\right)}{\partial \Delta \lambda_{(l)}}+\frac{1}{\alpha^{2}} \frac{\partial B_{x}^{(l)}\left(\Delta \lambda_{(l)}\right)}{\partial \Delta \lambda_{(l)}}\right. \\
& \left.\quad+\frac{1}{\beta^{2}} \frac{\partial B_{t}^{(l)}\left(\Delta \lambda_{(l)}\right)}{\partial \Delta \lambda_{(l)}}\right]+\sigma_{(l)}^{2}(t) N_{X_{\alpha \beta}}^{(l)}+v \frac{\hat{P}_{j}(t)}{\hat{P}_{l}(t)}\left[\hat{\lambda}^{(l)}(t)-\hat{\lambda}^{(j)}(t)\right], \\
& \hat{\lambda}^{(l)}\left(t_{0}\right)=\hat{\lambda}_{0}^{(l)},(l, j=\overline{1,2}, j \neq l), \\
& \hat{\lambda}(t)=\hat{P}_{1}(t) \hat{\lambda}^{(1)}(t)+\hat{P}_{2}(t) \hat{\lambda}^{(2)}(t),
\end{aligned}
$$

where:

$$
\begin{aligned}
& N_{X_{\alpha \beta}}^{(l)}=\frac{1}{2 C_{0}^{(l)}} \frac{\partial}{\partial \hat{\lambda}^{(l)}} \int_{-X}^{X}\left\{2 \eta^{(l)}(x, t) S^{(l)}\left(x-\hat{\lambda}^{(l)}, t\right)+\frac{1}{\alpha}\left[\frac{\partial \eta^{(l)}(x, t)}{\partial x} S^{(l)}\left(x-\hat{\lambda}^{(l)}, t\right)\right.\right. \\
& \left.+\eta^{(l)}(x, t) \frac{\partial S^{(l)}\left(x-\hat{\lambda}^{(l)}, t\right)}{\partial x}\right]+\frac{1}{\alpha^{2}} \frac{\partial \eta^{(l)}(x, t)}{\partial x} \frac{\partial S^{(l)}\left(x-\hat{\lambda}^{(l)}, t\right)}{\partial x} \\
& +\frac{1}{\beta}\left[\frac{\partial \eta^{(l)}(x, t)}{\partial t} S^{(l)}\left(x-\hat{\lambda}^{(l)}, t\right)+\eta^{(l)}(x, t) \frac{\partial S^{(l)}\left(x-\hat{\lambda}^{(l)}, t\right)}{\partial t}\right] \\
& \left.+\frac{1}{\beta^{2}} \frac{\partial \eta^{(l)}(x, t)}{\partial t} \frac{\partial S^{(l)}\left(x-\hat{\lambda}^{(l)}, t\right)}{\partial t}\right\} d x .
\end{aligned}
$$

(As a remark: in many cases the measurements signals and noises are (or are supposed to be) uncorrelated). In this solution, the spatial-time-varying signals are processed in parallel by two estimators exchanging information between them, each based (for example) upon a particular model of target dynamics intensity and adaptive expansion or contraction of the target tracker field of view attained by generating the probabilistically weighted average of the two filter state estimates.

The variance equation (Eq. (8)) is presented below:

$$
\begin{aligned}
& \frac{d \sigma_{(l)}^{2}(t)}{d t}=2 \sigma_{(l)}^{2}(t) \frac{\partial f^{(l)}\left(\hat{\lambda}^{(l)}, u, t\right)}{\partial \hat{\lambda}^{(l)}}+\frac{\sigma_{(l)}^{4}(t)}{2 C_{X}^{(l)}}\left[2 \frac{\partial^{2} B^{(l)}\left(\Delta \lambda_{(l)}\right)}{\partial \Delta \lambda_{(l)}^{2}}+\frac{1}{\alpha^{2}} \frac{\partial^{2} B_{X}^{(l)}\left(\Delta \lambda_{(l)}\right)}{\partial \Delta \lambda_{(l)}^{2}}\right. \\
& \left.\quad+\frac{1}{\beta^{2}} \frac{\partial^{2} B_{t}^{(l)}\left(\Delta \lambda_{(l)}\right)}{\partial \Delta \lambda_{(l)}^{2}}\right]+\sigma_{(l)}^{4}(t) N_{\alpha \beta}^{(l)^{*}}+q^{(l)}(t)+v \frac{\hat{P}_{j}(t)}{\hat{P}_{l}(t)}\left[\sigma_{(J)}^{2}(t)-\sigma_{(l)}^{2}(t)\right. \\
& \left.\quad+\left(\hat{\lambda}^{(j)}(t)-\hat{\lambda}^{(l)}(t)\right)^{2}\right], \quad \sigma_{(l)}^{2}\left(t_{0}\right), \quad(l, j=\overline{1,2}, \quad j \neq l), \\
& \sigma^{2}(t)=\hat{P}_{1}(t) \sigma_{(1)}^{2}(t)+\hat{P}_{2}(t) \sigma_{(2)}^{2}(t),
\end{aligned}
$$

where:

$$
\begin{aligned}
& N_{\alpha \beta}^{(l)}{ }^{*}=\frac{1}{C_{0}^{(l)}} \frac{\partial^{2}}{\partial\left(\hat{\lambda}^{(l)}\right)^{2}} \int_{-X}^{X}\left(\frac{1}{\alpha}\left[\frac{\partial \eta^{(l)}(x, t)}{\partial x} S^{(l)}\left(x-\hat{\lambda}^{(l)}, t\right)+\eta^{(l)}(x, t) \frac{\partial S^{(l)}\left(x-\hat{\lambda}^{(l)}, t\right)}{\partial x}\right]\right. \\
& +\frac{1}{\alpha^{2}} \frac{\partial \eta^{(l)}(x, t)}{\partial x} \frac{\partial S^{(l)}\left(x-\hat{\lambda}^{(l)}, t\right)}{\partial x}+\frac{1}{\beta}\left[\frac{\partial \eta^{(l)}(x, t)}{\partial t} S^{(l)}\left(x-\hat{\lambda}^{(l)}, t\right)\right. \\
& \left.\left.+\eta^{(l)}(x, t) \frac{\partial S^{(l)}\left(x-\hat{\lambda}^{(l)}, t\right)}{\partial t}\right]+\frac{1}{\beta^{2}} \frac{\partial \eta^{(l)}(x, t)}{\partial t} \frac{\partial S^{(l)}\left(x-\hat{\lambda}^{(l)}, t\right)}{\partial t}\right) d x .
\end{aligned}
$$

The variance equations (Eq. (8)) are the new Riccati-type differential equations derived 1) for 
systems with random structure 2) with cross correlation functions (their second derivatives) 3) for signal processing in STVGMCN.

The filtering algorithm for correlation-extremum systems with random structure for estimation of signal position along the $y$ axis has been derived, similarly, and for both components of the state vector $\lambda_{x}(t)$ and $\lambda_{y}(t)$ the appropriated adaptive estimation algorithm has been derived.

Signal processing in the presence of the STVGMCN is a more general form of the estimation problem, which allows one 1) to obtain the solution for the measurement STVGWN $[9,10]$, modifying the equations (Eqs. (6-8)), considering $\alpha \rightarrow \infty, \beta \rightarrow \infty$, and in this fashion maximizing the corresponding bandwidths, and 2) to receive the background model reflecting the real environmental conditions more adequately by changing the parameters of the STVGMCN $\alpha$ and $\beta$.

Using the proposed algorithms based on the systems with random structure theory there is no need in experimental or artificial tuning the gain matrix to avoid diverge as it was necessary for nonlinear filters in systems with a deterministic structure.

The obtained linearized solution of the derived algorithms allows to receive the a priori performance evaluation of the signal processing system in different conditions.

It can also be noticed that the new algorithms take advantages of recent increases in processor speeds satisfying the required computational burdens, and of the correlation-extremum signal processing properties.

\section{Conclusions}

The proposed new correlation-extremum algorithms for computing the a posteriori probabilities of states, the state estimates, and the variance, are obtained using the theory of Markov processes and stochastic systems with random structure for the adaptive estimation problem, when the state and the parameter models follow Markov processes, and the measurements are the nonlinear spatial-time-varying signals of different physical nature where the measurements noise is the spatial-time-varying Gaussian-Markov colored process first considered as a measurement noise model in systems with random structure to describe more adequately the environmental influences on SDS.

The proposed extension of the correlation-extremum methods to the theory of Markov processes and systems with random structure provides adaptive features for the combined system by generating the probabilistically weighted average of the state estimates, with the gain first as an analytical function (not as an experimentally or artificially modified value) depending on the spatial cross-correlation function derivatives and the a posteriori probabilities of states, and assures the complex SDS with random structure reliability and operation in varying and uncertain external conditions.

There are many potential military and civil application areas of the derived nonlinear spatialtime-varying signal processing algorithms, in particular in such nonlinear SDS as tracking, navigation systems, robotics equipped with image sensors (e.g., radar, optics, and etc.) using the spatial-time-varying measurements information of different nature fields.

\section{Acknowledgement}

The author would like to thank the mentioned authors [1-8], whose input researches generate the further output investigations as an innovative process.

\section{References}

[1] Kalman R. E. A new approach to linear filtering and prediction problems. Transactions on ASME, Journal of Basic Engineering, Vol. 82, 1960, p. 34-45.

[2] Knoll A., Edelstein M. Estimation of local vertical and orbital parameters for an Earth satellite using horizon sensor measurements. AIAA Journal, Vol. 3, Issue 2, 1965, p. 338-345. 
[3] Sage A. P., Melse J. L. Estimation Theory with Applications to Communication and Control. NewYork, McGraw-Hill, 1976, (in Russian).

[4] Chen Guanrong, Chui Charles K. A modified adaptive Kalman filter for real-time applications. IEEE Transactions on Aerospace and Electronic Systems, Vol. 27, Issue 1, 1991, p. 149-153.

[5] Cardillo G. P., Mrstic A. V., Plambeck T. A track filter for reentry objects with uncertain drag. IEEE Transactions on Aerospace and Electronic Systems, Vol. 35, Issue 2, 1999, p. 394-408.

[6] Maybeck P. S., Suizu R. I. Adaptive tracker field-of-view variation via multiple model filtering. IEEE Transactions on Aerospace and Electronic Systems, Vol. 21, Issue 4, 1985, p. 529-538.

[7] Bryson A. E., Johansen D. E. Linear filtering for time-varying systems using measurements containing colored noise. IEEE Transactions on Automatic Control, Vol. 10, 1965, p. 4-10.

[8] Baklitski V., Yuriev A. Correlation-Extremum Methods in Navigation. Radio and Communication, Moscow, 1982, (in Russian).

[9] Kolosovskaya T. Spatial-time-varying signals processing algorithms in systems with random structure. Mechanical Engineering and Machine Reliability Problems, Vol. 5, 1995, p. 105-112, (in Russian).

[10] Kolosovskaya T. Nonlinear filtering and identification algorithms for correlation-extremum dynamic systems with random structure. Journal of Vibroengineering, Vol. 8, 2016, p. 531-537.

[11] Kolosovskaya T. Adaptive estimation using linearized spatial-time-varying signal processing algorithms in systems with random structure. 15th International Conference on Aviation and Cosmonautics, Moscow, 2016, p. 450-452. 